\title{
Erratum: $\boldsymbol{\eta}-\eta^{\prime}$ mixing and the derivative of the topological susceptibility at zero momentum transfer [Phys. Rev. D 95, 033001 (2017)]
}

\author{
N. F. Nasrallah \\ (Received 4 June 2018; published 21 June 2018)
}

DOI: 10.1103/PhysRevD.97.119901

My article " $\eta-\eta$ ' mixing and the derivative of the topological susceptibility at zero momentum transfer, Phys. Rev. D 95, 033001 (2017) contains several misprints and errors resulting from sign mistakes

1. In Sec. II: the right-hand side of the second of Eq. (2.3), $\sqrt{2 / 3} Q$ should be replaced by $2 \sqrt{6} Q$ and in the line after change the definition of $\mathrm{Q}$ to $Q=\frac{\alpha_{s}}{8 \pi} G \tilde{G}$.

2. In Sec. III: In Eq. (3.6) the signs inside both brackets should be + :

$$
\begin{aligned}
\langle 0|Q| \eta\rangle & =\sqrt{\frac{1}{12}}\left(f_{8 \eta}+\sqrt{2} f_{0 \eta}\right) m_{\eta}^{2} \\
\left\langle 0|Q| \eta^{\prime}\right\rangle & =\sqrt{\frac{1}{12}}\left(f_{8 \eta}+\sqrt{2} f_{0 \eta^{\prime}}\right) m_{\eta^{\prime}}^{2}
\end{aligned}
$$

In Eq. (3.8): $C_{21}=-\left(\frac{\alpha_{s}}{8 \pi}\right)^{2} \frac{2}{\pi^{2}}\left(1+\frac{83}{4} \frac{\alpha_{s}}{\pi}\right)$ and $C_{-1}=\frac{1}{8}\left(\frac{\alpha_{s}}{\pi}\right)\left\langle g_{s} \frac{\alpha_{s}}{\pi} G^{3}\right\rangle$.

In Eq. (3.11): In the second term on the right-hand side 1.30 should be replaced by 2.31 :

$$
\chi^{\prime}(0)=a_{1} \chi(0)+2.31 \times 10^{-3} \mathrm{GeV}^{2}+\delta_{2}
$$

3. After Eq. (3.14) the text in the rest of Sec. III should be replaced by:

In order to proceed further the constant $c$ has to be determined. This is done by considering the integral $\frac{1}{2 \pi i} \int_{c} d t p(t) \chi(t)$ because the only poles of the integrand lie at the pseudoscalars we have

$$
0=\langle 0|Q| \pi\rangle^{2}+\langle 0|Q| \eta\rangle^{2} p\left(m_{\eta}^{2}\right)+\left\langle 0|Q| \eta^{\prime}\right\rangle^{2} p\left(m_{\eta^{\prime}}^{2}\right)+\delta_{0}
$$

with

$$
\delta_{0}=\frac{1}{2 \pi i} \int_{c} d t p(t) I(t)=\frac{c}{2 \pi i} \int_{c} d t p(t) t^{2} K_{2}^{2}\left(\rho_{c} \sqrt{-t}\right) .
$$

Asymptotic forms of $K_{2}(x)$ are given in Dwight [20] these are used to evaluate the integral above which yields

$$
c=-.376 \times 10^{-3} \text {. }
$$

This together with a similar evaluation of the corresponding integrals appearing in the expressions of $\delta_{1}$ and $\delta_{2}$ give

$$
\delta_{1}=.177 \times 10^{-3} \mathrm{GeV}^{4} \text { and } \delta_{2}=.028 \times 10^{-3} \mathrm{GeV}^{6}
$$

which corresponds to

$$
\chi(0)=1.10 \times 10^{-3} \mathrm{GeV}^{4} \text { and } \chi^{\prime}(0)=3.5 \times 10^{-3} \mathrm{GeV}^{2}
$$

The value obtained for $\chi(0)$ is quite close to the one computed on the lattice [21] $\chi(0)=1.33 \times 10^{-3} \mathrm{GeV}^{4}$ and to the one given by the Witten-Veneziano [22,23] formula obtained in the large $N_{c}$ limit 


$$
\chi(0)=\frac{f_{\pi}^{2}}{2 n_{f}}\left(m_{\eta}^{2}+m_{\eta^{\prime}}^{2}-2 m_{K}^{2}\right)=1.05 \times 10^{-3} \mathrm{GeV}^{4}
$$

As to $\chi^{\prime}(0)$ the value obtained is relatively large, close to the one advocated by Ioffe [12], $\chi^{\prime}(0)=$ $(2.9 \pm .4) \times 10^{-3} \mathrm{GeV}^{2}$.

4. In Sec. IV: $\chi^{\prime}(0)=3.5 \times 10^{-3} \mathrm{GeV}^{2}$.

The result for the couplings, mixing angles and masses are unchanged. The final results for the topological susceptibility and its derivative at the origin, Eq. (3.19), differ considerably from the ones in the original article. 